MATEC Web of Conferences 22,01004 (2015)

DOI: $10.1051 /$ matec conf/ 20152201004

(C) Owned by the authors, published by EDP Sciences, 2015

\title{
A Study on the Influence of Electromagnetic Radiation on Nervous Sys- tem
}

\author{
Xinlin Dong, Lihua Niu, Dongjie Wang \& Yajing He \\ Center Lab. of Langfang Health Vocational College, Langfang, Hebei, China
}

\begin{abstract}
Being applied widely, electromagnetic wave is closely related with our life. But this material has brought pollutions to the environment as well as influences on functions of organisms. In order to explore the influence of electromagnetic radiation on nervous system, this paper takes adult mice on 35th day as research objects, designs a water maze experiment and explores features of escaping latency of mice in the control group and in the group with radiation. In this research, methods of building a GHz TEM cell and a simulation model of mouse head with AutoCAD 2010 and XFDTD are provided, verifying that the simulation model meets the needs of the experiment. It concludes that the electromagnetic radiation causes memory deterioration of mice, and exerts its certain influence on nervous system.
\end{abstract}

Keywords: finite-difference time-domain method; electromagnetic radiation; XFDTD software; simulation model; water maze experiment

\section{INTRODUCTION}

The development of industrial and information technologies drives a rapid growth of electromagnetic technology at the current stage. The electromagnetic technology occupies a large part of fields in our life, such as broadcast, communication, home appliances and so on. These articles for daily use cause electromagnetic pollution in people's life. Reisberg B, et al. (1982) formulated the safety limit of electromagnetic radiation ${ }^{[1]}$ and carried out fixed-point monitoring in line with the safety limit. Results prove that the electromagnetic pollution is becoming more and more serious in the living environment and needs to be urgently solved ${ }^{[2]}$.

Researches indicate that ${ }^{[3]}$ : neurons are sensitive to electromagnetic radiation, which can lead to the change and functional damage of neural synapse structure. And neuron damage is closely related to learning and memory function. Ingram EM, et al. (2002) indicates that the electromagnetic radiation can cause intracellular calcium overload ${ }^{[4]}$. Li Li (2012) points out that the electromagnetic radiation can lead to dysfunction of multiple organs and systems. Long-term electromagnetic radiation would cause damages to a variety of neurobehavioral functions including learning and memory function ${ }^{[5]}$. An early performance of central nervous system injury caused by electromagnetic radiation is neurobehavioral dysfunction ${ }^{[6]}$. People permanently working in the environment of electromagnetic field will have symptoms such as dizziness, headache, fatigue, memory deterioration, sleep disorders and so on. A major performance is the obstacle of learning and memory function. And it is also a high-risk factor to suffer from Alzheimer's disease ${ }^{[7]}$.
Many people have made efforts in researches about electromagnetic radiation's influence on functions of organism. In recent years, theoretical methods proposed by scholars have made a great contribution to studies on shielding electromagnetic radiation. Hongmei Zhou ${ }^{[8]}$, et al. (2009) calculates specific absorption rate and distribution of human body simulation model with FDTD method and concludes that brain, cerebellum and spinal cord in a central nervous system have high absorption of electromagnetic energy at different frequencies. Guobing $\mathrm{Lu}^{[9]}$, et al. (2013) points out that the electromagnetic radiation of $2000 \mu \mathrm{W} / \mathrm{cm}^{2}$ can lead to the memory function deterioration of mice. This mechanism may be related to the expression decline of the hippocampus NR2B protein and mRNA of mice. Yanzhong $\mathrm{Li}^{[10]}$, et al. (2013) indicates that electromagnetic radiation can cause changes of body physiological indicators. It has certain effects on body health, which will aggravate with the working years in radiation. Ahui Dai ${ }^{[11]}$, et al. (2014) states that electromagnetic radiation can directly or indirectly activate the NADPH oxidase complex, which then transfers intracellular NADPH electrons to form active oxygen or involves in life process such as inflammation defense and tissue repair through a series of inflammatory factors and relevant matrix metalloproteinase. Qi Zhang ${ }^{[12]}$, et al. (2014) indicates that environments of high electromagnetic radiation have certain influence on children's neurobehavioral functions. Both Xinxing Yang ${ }^{[13]}$, et al (2014) and Fang Cheng ${ }^{[14]}$ (2015) point out that people's health can be protected by preventing and reducing harms of electromagnetic radiation.

Based on previous studies, a GHz TEM cell and a 3D electromagnetic model of mouse head are built up with AutoCAD 2010 and XFDTD, and the influence 
of electromagnetic radiation on nervous system is verified by the water maze experiment so as to provide a theoretical basis for the prevention and control of electromagnetic radiation.

\section{BASIC THEORY OF 3D ELECTROMAGNETIC RADIATION BASED ON XFDTD}

Electromagnetic wave is produced by an appropriate vibration source, generating the vortex electric field by varying magnetic field and generating the vortex magnetic field by electrochemical electric field. The wave transmitted by electromagnetic oscillation in a space or a material is actually the process of transferring electromagnetic energy, namely a transverse wave. The propagation velocity of electromagnetic wave is the light speed, the propagation law of which satisfies Maxwell's equations. It includes five waves: the long wave, the medium wave, the short wave, the ultra-short wave and the microwave ${ }^{[15]}$. The calculation of energy $W$ and momentum density $g$ of electromagnetic wave is shown in Formula (1). $E$ stands for electromagnetic intensity, $H$ is magnetic field intensity, $\mu$ is permeability, $\varepsilon$ is permittivity and $C$ is capacitance ${ }^{[16]}$

Electromagnetic radiation is a phenomenon that the electromagnetic energy is emitted from a radiation source to the space in the form of electromagnetic wave. The principle of simulating the 3D electromagnetic radiation with XFDTD (3D full-wave electromagnetic simulation software developed by U.S. REMCOM company) is the electromagnetic numerical calculation method FDTD (finite-difference time-domain) ${ }^{[17]}$.

$$
\left\{\begin{array}{l}
W=E H \sqrt{\mu \varepsilon} \\
g=\frac{E H}{C^{2}}
\end{array}\right.
$$

XFDTD software has the following features:

1) The function of importing and exporting of formats like DXF and SAT. It also has the import interface for advanced 3D model files like PROE, Inventor, IGES, Catia, STEP and so on.

2) It has a superior performance in material processing with unique simulation features of biological electromagnetism.

3) It automatically or manually generates grid with adaptive grid functions.

4) It supports distributed computation and multiple CPU parallel computation.

5) It is applicable to frequency domain and time domain analysis. Through the analysis on time domain, the simulation results of broadband can be obtained in one time with high efficiency of simulation and accurate simulation results.

6) Visual results: planar electric and magnetic fields of steady state, planar current distribution, planar SAR value, 3D color distribution diagram of steady-state surface current, output MPEG image, Smith image, and Fourier Transform of transient results.

\subsection{Principle of FDTD}

Based on difference equations, the finite-difference time-domain method ${ }^{[18]}$ converts the rotating equation in Maxwell's equations that contains the most basic rules of electromagnetic field into differential equations ${ }^{[19]}$ and calculates electromagnetic field through spatial dispersion called Yee Grid ${ }^{[20]}$ in a 4D space including time. Usually, the calculation of time-domain electromagnetic field should be conducted in a 4D space including time and the key of converting a Maxwell's rotating equation into a differential radical is to rationally disperse six unknown fields in a $4 \mathrm{D}$ space. If there is an active domain, the parameters of involved media will be isotropic and do not be changed with time. There might be electric and magnetic loss with electromagnetic field problems. The rotating equation in Maxwell's equations can be expressed as Formula (2). In this formula, $E$ is electric field intensity $(\mathrm{V} / \mathrm{m}), H$ is magnetic field intensity $(\mathrm{A} / \mathrm{m}), \quad \varepsilon$ is permittivity $(\mathrm{F} / \mathrm{m}), \sigma$ is conductivity $(\mathrm{S} / \mathrm{m})$ and ${ }^{\sigma} m$ is permeability $(\Omega / \mathrm{m})$ :

$$
\left\{\begin{array}{c}
\nabla \times E=-\mu \frac{\partial H}{\partial t}-\sigma_{m} H \\
\nabla \times H=\varepsilon \frac{\partial E}{\partial t}+\sigma E
\end{array}\right.
$$

As shown in Figure 1, the grid system of Yee is presented with a rectangular coordinate system. The feature is the spatial points in each component of electric field and magnetic field that are crosswise placed. In the same coordinate plane, one magnetic field component is surrounded by the electric field and the electric field component is surrounded by the magnetic field component. In such space settings, the fixed quantity of electromagnetic wave transmitted in space can be obtained through Ampere circular current law and Faraday electromagnetic induction law.

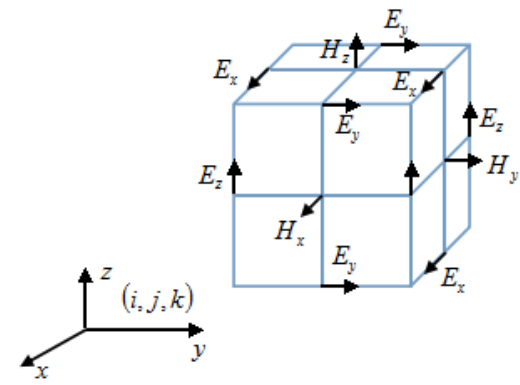

Figure 1. Yee grid in a rectangular coordinate system

In Figure $1, E_{x}, E_{y}, E_{z}, H_{x}, H_{y}, H_{z}$ respectively 
stands for a partial derivative. If the above six partial derivatives respectively solves the partial derivative of $t$, the grid spacing in three coordinate axes can be expressed with $\Delta x, \Delta y, \Delta z$ respectively. Integers $i, j, k$ are used to stand for grid number or grid point label of $x$-axis, $y$-axis and $z$-axis. Thus, the space coordinates of any grid point can be expressed as $(i, j, k)=(i \Delta x, j \Delta y, k \Delta z)$.

Time-step length is expressed by $\Delta t, n$ is time steps expressed by nonnegative integers, and $f(x, y, z, t)$ means that it is related not only with space coordinates but also with time variables. The relationship is shown in Formula (3):

$$
F^{\prime \prime}(i, j, k)=F(i \Delta x, j \Delta y, k \Delta z, n \Delta t)
$$

\subsection{Numerical dispersion}

If the spatial medium characteristics of electromagnetic wave are related to frequency, the propagation velocity of electromagnetic wave is also a function of frequency. This phenomenon is called dispersion and the medium in the phenomenon is called dispersive medium. Apparently, the propagation velocity of electromagnetic wave has nothing to do with frequency in non-dispersive media.

Dispersion can be also produced in Yee grid space, which means that dispersion can also exist in non-dispersive media. This non-physical dispersion is defined as numerical dispersion, which may cause pulse waveform distortion and artificial isotropic and false refraction phenomena. Therefore, in a finite-difference time-domain method, the numerical dispersion is a factor that must be considered as well as an important factor that can improve calculation precision ${ }^{[21]}$. The basic method of analyzing numerical dispersion is to import the general form of a monochromatic plane wave into a difference equation so as to obtain the relationship among frequency, spatial-step length and time-spatial length. As the TM wave is the objective in this research, the numerical dispersion relation in a $3 \mathrm{D}$ space can be expressed by Formula (4). In this formula, $\Delta t$ is time-step length, $\Delta x, \Delta y, \Delta z$ respectively stands for the spatial-step length along $x$-axis, $y$-axis and $z$-axis. The component of wave vector $\mathrm{K}$ is presented by $k_{x}, k_{y}, k_{z}, \omega$ is angular frequency and $v$ is light speed:

$\left(\frac{1}{v \Delta t}\right)^{2} \sin ^{2} \frac{\omega \Delta t}{2}=\frac{1}{(\Delta x)^{2}} \sin ^{2} \frac{k_{x} \Delta x}{2}+\frac{1}{(\Delta y)^{2}} \sin ^{2} \frac{k_{y} \Delta y}{2}+\frac{1}{(\Delta z)^{2}} \sin ^{2} \frac{k_{z} \Delta z}{2}$

When $\Delta t$ and $\Delta x, \Delta y, \Delta z$ in the above formula all approach zero, the formula becomes an ideal dispersion relationship shown in Formula (5). It means that difference approximation causes numerical dispersion by replacing continuous derivatives. Therefore, numerical dispersion can be infinitely decreased by reducing time-step length and spatial-step length. How- ever, the reduction of time-step length and spatial-step length enlarges the grid space and increases the total grid number of FDTD method. Meanwhile, the requirement of computer storage space and computational time are also increased. Thus, numerical dispersion can be only decreased in FDTD method:

$$
\frac{\omega^{2}}{v^{2}}=k_{x}^{2}+k_{y}^{2}+k_{z}^{2}
$$

In the $3 \mathrm{D}$ grid space of FDTD, uniform cubic grids can be selected and set $k_{x}=k_{y}=k_{z}=k / \sqrt{3}$, namely the diagonal direction of electromagnetic wave propagation along the grid. Besides, an ideal dispersion relation can be also obtained by $\Delta s=v \sqrt{3} \Delta t$.

\section{ESTABLISHMENT OF THE SIMULATION MODEL OF ELECTROMAGNETIC RADIA- TION}

With a GHz TEM cell as the objective in this research, this paper first designs the 3D model by AutoCAD2010 software, imports SAT files into XFDTD software and installs the 3D electromagnetic model of the GHz TEM cell correctly so as to provide an accurate $3 \mathrm{D}$ field intensity map containing all places of the $\mathrm{GHz}$ TEM cell with XFDTD software, and the principle of which is the finite-difference time-domain method. Second, this paper carries out practical measurement with signal generator, RF power amplifier, EMC spectrum analyzer and measuring probe to make comparisons with simulation data.

\subsection{Establishment of the electromagnetic model in the GHz TEM cell}

The length unit of the model established by AutoCAD2010 is millimeter. Place a foam block at the bottom of the working area of the GHz TEM cell and a glass box at the top. These two centers are aligned with the cross intersection point of the working area. The two symmetric lines and the symmetric line in the plan view of Figure 2 are in the same plane. Side A of the glass box is on the right side and side $B$ is on the left side of the plan view. The 3D model of the $\mathrm{GHz}$ TEM cell can be obtained after install an experimental apparatus.

Steps of establishing the experimental electromagnetic model are presented as follows:

STEP1. After the selection of all components of AutoCAD 2010 window, we choose "file" in the menu bar and name the "outputted" SAT file as an "Experimental model".

STEP2. Start XFDTD software and import "Experimental model". Name each component link in the $\mathrm{GHz}$ TEM cell when import SAT files and set corresponding relative dielectric constant, conductivity and colors. Settings of each part are shown in Table 1:

STEP3. In XFDTD, respectively assign relevant 


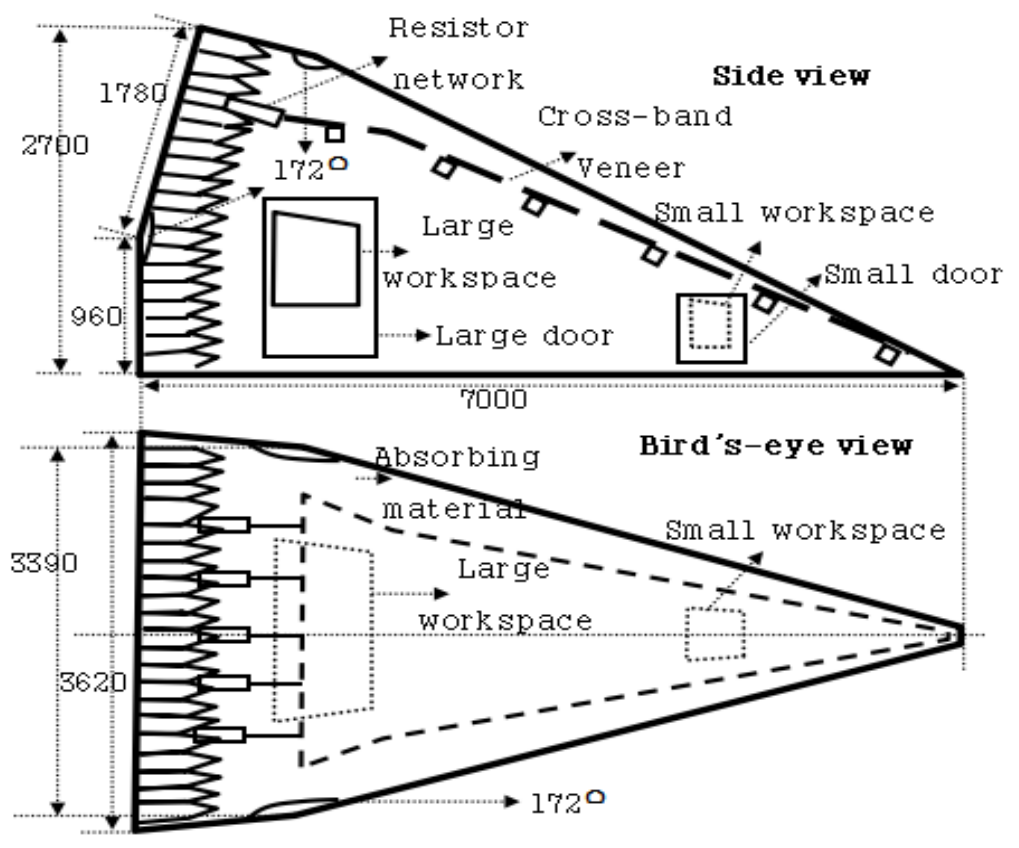

Figure 2. Diagram of the structure and size of the TEM cell

Table1. Settings in each component link of the TEM cell

\begin{tabular}{lccc}
\hline Names & Relative dielectric constant & Conductivity (S/m) & Colors \\
\hline Core board & 3 & Good conductor (system default) \\
Horizontal supporting bar & & 0 & Sky blue \\
Shell of GTEM Cell & 5.5 & Good conductor (system default) & Red \\
Glass box & 1 & $1.83 \mathrm{e}+007$ & Gray \\
Resistance & & $2-\mathrm{j} 1$ & Blue \\
Absorbing material & 3 & 0 & Sky blue \\
Plastic rods & 2 & 0 & Purple \\
Board and disk & 2.6 & Light red \\
Foam block & & & 0
\end{tabular}

parameters to component parts (click the button "Apply Material") and then import parameters into

which is in line with the $3 \mathrm{D}$ model built by AutoCAD 2010. Thus, the experimental electromagnetic model can be successfully established.

\subsection{Grid establishment and operating parameters setting}

After the establishment of the electromagnetic model, a geometric object can be only used to present grid by dispersion of FDTD and conduct the calculation of FDTD. The size of grid of FDTD is influenced by
XFDTD. The imported length unit is millimeter,

wave length. In FDTD, the size of grid must be smaller or equal to one tenth of the minimum wave length. So, the grid size is restrained by Formula $(6)^{[17]}$. Here, $\Delta x_{\max }$ is the maximum size of FDTD grid, $f$ is the frequency $(\mathrm{Hz}), \quad c$ is the light speed $\left(3 \times 10^{8} \mathrm{~m} / \mathrm{s}\right)$. When $f=1800 \mathrm{MHz}, \Delta x_{\max }=16.67 \mathrm{~mm}$ is the largest grid size:

$$
\Delta x_{\max }=\frac{c}{10 \times f}
$$


This experiment is conducted based on such parameters: $\mathrm{t}$ frequency of $1.8 \mathrm{GHz}$, computer main frequency of $2.45 \mathrm{G}$ and $8 \mathrm{G}$ of internal storage. The grid can be set as a uniform grid, the size (length, width, height) of which is $13 \mathrm{~mm} \times 13 \mathrm{~mm} \times 13 \mathrm{~mm}$ and the grid type is an electric field pattern with the combination of factors as each link size of the GHz TEM cell and numerical dispersion. The GHz TEM cell is divided into $622 \times 301 \times 248$ grids, which are displayed in the Geometry-->Mesh window. In the Geometry-->View-->Mesh Mode window, adjust the Slice size and window views of coordinate planes of $X Y$, $\mathrm{YZ}$ and $\mathrm{ZX}$

In the Run Parameters-->Waveform window of XFDTD, we set the Type as Sinusoid, the Far-zone Transformation as Steady-State, the Waveform Frequency $\mathrm{GHz}$ as 1.8 , the Automatic Convergence as "Positive", and the Convergence Threshold as -30dB. It is a setting of excitation source that Discrete is set in the window Run Parameters --> Sources.

In the window Geometry-->View, select the border of the $3 \mathrm{D}$ coordinates $(\mathrm{X}=588, \mathrm{Y}=152, \mathrm{Z}=26)$ grid in the ZX plane when Slice $=158$. Click the right button and select Edit Port, the software will automatically skip to the Run-time Parameters-->Component / Ports window. Then select Static Voltages, open the setting window, and fill in $\mathrm{X}=588, \mathrm{Y}=152, \mathrm{Z}=26$. The steady state voltage is $15.4 \mathrm{~V}$. Click the Add and then the OK, select the coordinates $X=575, Y=152, Z=22$ in the same plane. And then the steady state voltage is $0 \mathrm{~V}$, the core board and the shell form a loop through a resistor network. In windows of Run Parameters--> Outer Boundary and Request Result-->Save Field Snapshot, we set Time Step and relevant parameters, set Location as the XY plane, and set Slice as snapshots of layer 10,18, 19, 20, 30 and 40. The Beginning of Time Step is 1, the Ending of Time Step is 10000 , and the Increment is 100 . The unit of time-step length is s. In the interface produced by clicking Add Sequence, we set relevant parameters in the Request Results-->Save Steady State window and select the $\mathrm{XY}$ plane. Set Type of Date as CEF and Slice as layer $10,18,19,20,30,40$, and select added planes. Before the simulation of XFDTD software, we select Save the Geometry and Save The Project on the top left corner of the software.

\subsection{Establishment of organism simulation model}

Mice on $7^{\text {th }}$ days are taken as objectives of research in this paper. Living organisms studied in this paper are put in the working area of the GHz TEM cell and then the electromagnetic model is established for simulation so as to calculate the specific absorption rate of mice head. In computational electromagnetism, this paper use the specific absorption rate (SAR) to accurately measure the electric field of biological tissues in electromagnetic radiation. SAR is defined as the energy absorbed by a unit mass in a biological tissue. As shown in Formula (7), $W$ is the absorbed electro- magnetic wave energy, $m$ is the mass of a living organism:

$$
S A R=\frac{W}{m}
$$

The 3D model of mice head is shown in Figure 3. The electromagnetic model can be established with AutoCAD2010 and XFDTD in a similar way. Export the 3D electromagnetic model as a file of SAT format and import it into XFDTD software. Set the relative dielectric constant and the conductivity in each part.

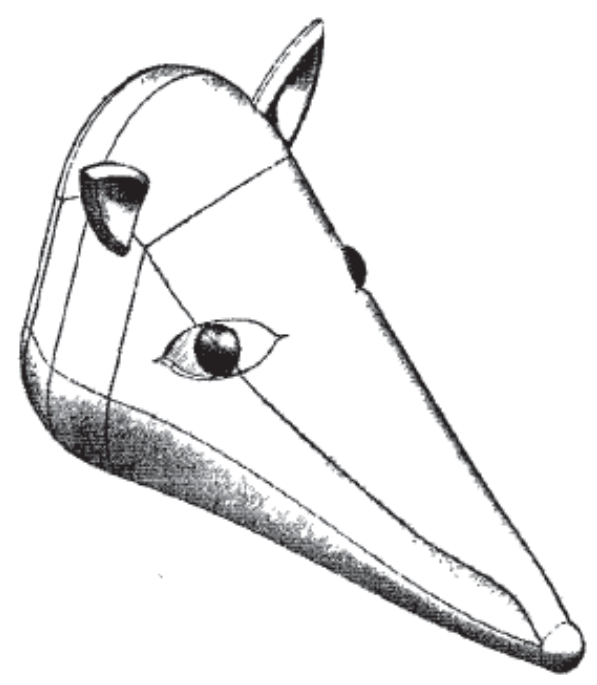

Figure3. 3D model of mice head

Relative dielectric constant, conductivity and color in each part of the 3D electromagnetic model of mice head are shown in Table 2. In parameters setting, the grid is uniform with the size of (length $7 \mathrm{~mm} \times$ width $7 \mathrm{~mm} \times$ height $7 \mathrm{~mm}$ ). The experimental model is divided into $641 \times 351 \times 288$ grids. Select the reservation of SAR in "Save Steady-State Data", the distribution diagram of SAR can be obtained by simulation. Select the plane $\mathrm{Y}=133$ and SAR can be obtained by moving the mouse. For example, the grid coordinate [171, 133, 18] stands for a point of the skin and $\mathrm{SAR}=0.676 \mathrm{~mW} / \mathrm{kg}$. The grid coordinate $[172,133,18]$ stands for a point of the bone and SAR $=0.290 \mathrm{~mW} / \mathrm{kg}$. The grid coordinate $[173,133,18]$ stands for a point of the brain and SAR $=0.254 \mathrm{~mW} / \mathrm{kg}$.

\section{SIMULATION RESULTS ANALYSIS AND MEDICAL EXPERIMENT ANALYSIS}

\subsection{Simulation results analysis}

After the establishment of the GHz TEM cell model, we select Run Calculation in the Result window of XFDTD software. The relevant uploading parameters and the speed of calculation are displayed in the window. The field strength value can be clearly observed 
MATEC Web of Conferences

Table 2. Settings in each link of the electromagnetic model of mice head

\begin{tabular}{llcc}
\hline Names & Relative dielectric constant & Conductivity (S/m) & Colors \\
\hline Mouse skin & 57.03 & 1.840 & Light red \\
Mouse bone & 11.93 & 0.302 & White \\
Mouse brain & 47.79 & 1.525 & Reddish brown \\
Horizontal supporting bar & 3 & 0 & Sky blue \\
Core board & & Good conductor (system default) & Light red \\
Foam block & 2.6 & 0 & Good conductor (system default) \\
Shell of GTEM Cell & & 0 & Red \\
Glass box & 5.5 & &
\end{tabular}

Table 3. CEF of sampling grids in Figure 4 (Unit: $\mathrm{dB} \mu \mathrm{V} / \mathrm{m}$ )

\begin{tabular}{llllllll}
\hline Sampling points & 1 & 2 & 3 & 4 & 5 & 6 & 7 \\
\hline A (simulation) & 147.1 & 147.6 & 147.9 & 147.2 & 147.0 & 147.5 & 147.2 \\
A (practical) & 147.3 & 147.5 & 147.4 & 147.4 & 147.2 & 147.1 & 147.0 \\
B (simulation) & 147.3 & 147.6 & 148.0 & 147.9 & 147.5 & 147.8 & 147.3 \\
B (practical) & 147.6 & 147.7 & 147.6 & 147.9 & 147.5 & 147.8 & 147.3 \\
C (simulation) & 147.8 & 147.9 & 148.0 & 148.0 & 147.5 & 148.2 & 148.1 \\
C (practical) & 147.8 & 147.9 & 147.7 & 148.3 & 147.8 & 148.2 & 148.3 \\
D (simulation) & 148.3 & 148.3 & 148.5 & 148.2 & 148.7 & 149.0 & 149.1 \\
D (practical) & 148.5 & 148.3 & 148.0 & 148.4 & 148.3 & 149.0 & 149.1 \\
E (simulation) & 148.9 & 148.9 & 149.0 & 148.9 & 149.0 & 150.0 & 149.3 \\
E (practical) & 148.8 & 149.0 & 149.0 & 148.9 & 149.0 & 149.9 & 149.1 \\
F (simulation) & 149.2 & 150.0 & 149.2 & 149.1 & 149.1 & 149.3 & 149.4 \\
F (practical) & 149.4 & 149.3 & 149.2 & 149.2 & 149.4 & 150.0 & 149.8 \\
G (simulation) & 149.5 & 150.2 & 149.2 & 149.2 & 149.8 & 150.1 & 150.2 \\
G (practical) & 149.7 & 150.2 & 149.6 & 149.3 & 149.8 & 150.1 & 150.2
\end{tabular}

by obtaining the calculated results and moving the mouse on to the result picture acquired by CEF (effective value). The field strength value can be observed at the bottom right corner of the window of XFDTD software. In this experiment, the area for experimental objectives is $Z=18$, the grid scale of $X$ is $[445,476]$ and the grid scale of $\mathrm{Y}$ is $[139,164]$. The grid equivalent figure is shown in Figure 4.

In Figure 4, black grids are sampling grids. Letter A, $\mathrm{B}, \mathrm{C}, \mathrm{D}, \mathrm{E}, \mathrm{F}$, and $\mathrm{G}$ on the $y$-axis respectively stands for the $1^{\text {st }}$ line, the $2^{\text {nd }}$ line, the $3^{\text {rd }}$ line, the $4^{\text {th }}$ line, the $5^{\text {th }}$ line, the $6^{\text {th }}$ line and the $7^{\text {th }}$ line of the sampling grids. Number $1,2,3,4,5,6,7$ respectively stands for the $1^{\text {st }}$ column, the $2^{\text {nd }}$ column, the $3^{\text {rd }}$ column, the $4^{\text {th }}$ column, the $5^{\text {th }}$ column, the $6^{\text {th }}$ column and the $7^{\text {th }}$ column of the sampling grids. For example, A1 stands for $(X=445, Y=139, Z=18), B 2$ stands for $(X=450$,

\section{$\mathrm{Y}=163, \mathrm{Z}=18)$ and so on}

Simulation field strength values of sampling points of the GHz TEM cell model are presented in Table 3, which are compared with the practical measured value when the frequency is $1.8 \mathrm{GHz}$. Instruments for practical measurement include: Agilent E8648C signal generator ${ }^{[22]}$, Agilent E7402A EMC spectrum analyzer, GLG25 RF power amplifier and HZ-11 904 measuring probe. At the beginning of practical measurement, we start the signal generator with the frequency of $1.8 \mathrm{GHz}$ and output level of $101 \mathrm{~dB} \mu \mathrm{V} / \mathrm{m}$. And the practical measured value of field strength can be obtained by connecting the RF power amplifier to the working area of the GHz TEM cell.

It can be known from Table 3 that the field strength value increases along the $x$-axis direction and the selected grids gradually approach the core board. The 


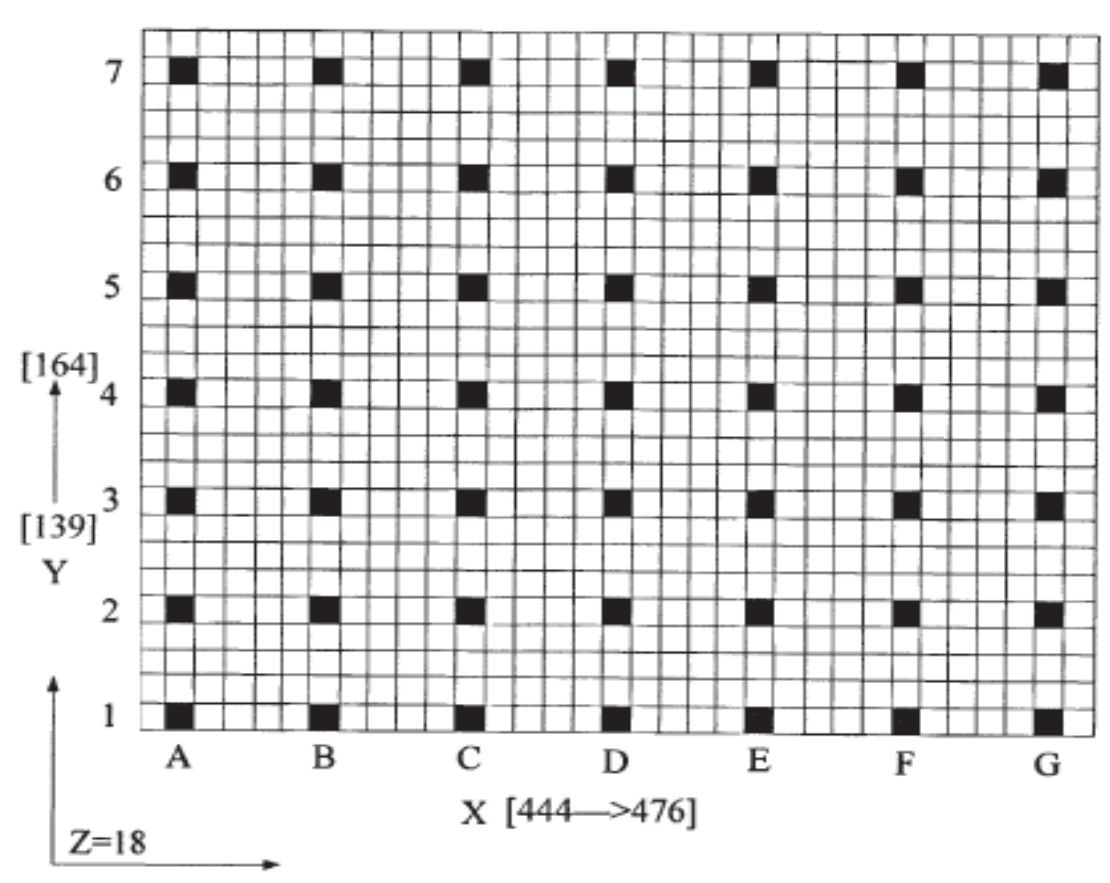

Figure 4. Equivalent figure of grids

Table 4. Measured results of the water maze experiment

\begin{tabular}{lllll}
\hline Groups & The $1^{\text {st }}$ day & The $2^{\text {nd }}$ day & The $3^{\text {rd }}$ day & The $4^{\text {th }}$ day \\
\hline Group A & $54.616 \pm 16.473$ & $57.058 \pm 16.224$ & $62.225 \pm 12.946$ & $67.291 \pm 21.568$ \\
Group B & $33.908 \pm 9.411$ & $38.350 \pm 9.075$ & $38.541 \pm 10.464$ & $38.691 \pm 11.477$ \\
$P$ & 0.01 & 0.02 & $<0.001$ & 0.01 \\
\hline
\end{tabular}

change along the direction of $y$-axis is quite small. Sampling grids on the $y$-axis and the core board are parallel. From this data, the field strength distribution in the experimental area is uniform. There are certain

\subsection{Water maze experiment analysis}

Experimental subject: We choose some clean mice with $4-5 \mathrm{~g}$ on the $35^{\text {th }}$ days based on the proportion of male and female which is $1: 1$. Those 24 mice are randomly divided into two groups: the group A with radiation and the controlled group B. And each group is with 12 mice.

Experimental method: We simulate the electromagnetic radiation environment of mobile phone by the TEM cell. The radiation frequency is $1800 \mathrm{MHz}$ and the field strength is $148.9 \mathrm{~dB} \mu \mathrm{V} / \mathrm{m}$. According to the experimental design, we respectively carry out the water maze experiment to test learning and memory ability of mice after radiation treatment in group A and $\mathrm{B}$, and compare the difference of two groups with statistical methods. The water maze instrument consists of two parts: a circular pool and an automatic errors between the practical value and the simulative value, but the error range is controlled within $\pm 2 \mathrm{~dB}$. It can be seen that the field strength in working area satisfies the experimental requirements.

video and analyzing system. A video camera is connected to the computer above the pool. When the current training time ends, the computer will stop tracking and recording the swimming track. As we mix the clean water and the milk powder in the pool, it becomes milk white without transparency. The platform is $5 \mathrm{~cm}$ above the pool. And the water temperature is controlled within $18^{\circ} \mathrm{C} \sim 20^{\circ} \mathrm{C}$. Mice are put in the water once a day from different quadrants. If an adult mouse cannot find the platform in $120 \mathrm{~s}$, it will be manually taken to the platform for $10 \mathrm{~s}$. If an adult mouse can find the platform in $120 \mathrm{~s}$, it will be allowed to stay at the platform for $10 \mathrm{~s}$ as well. And then we will dry the mice and take them back to the cage after training. After 3 days' training, we start to record the time for mice from being put into the pool to finding the platform for 4 days. 
Experimental objective: Determine the influence of electromagnetic radiation on nervous system of mice.

Experimental appliances: distilled water, milk powder, Morris water maze ${ }^{[24}$, SM22 automatic controller of water maze.

Measured data of the water maze experiment are presented in Table 4:

It can be known from the experimental results in Table 4 that the escaping latency of mice in two groups becomes shorter and shorter with the increase of training days. It reflects that adult mice in both groups begin to learn how to find the platform during training. But there are obvious differences in terms of learning and memorizing ability. The escaping latency of group A is obviously longer than that of group B. Obvious differences between those two groups are of statistical meanings. Therefore, the electromagnetic radiation has an influence on the memory of mice according to the aspect that electromagnetic radiation has a significant influence on nervous system.

\section{CONCLUSION}

This paper establishes a GHz TEM cell and an electromagnetic model of mouse head based on the analysis on FDTD principle and numerical dispersion principle, verifies the influence of electromagnetic radiation on nervous system by analyzing the rationality of establishing a simulation model, and draws conclusions shown as follows:

1) An accurate 3D field intensity map including all places of the GHz TEM cell that can be provided with the XFDTD software, the principle of which is the finite-difference time-domain method.

2) The small working area of GHz TEM cell can provide satisfactory electromagnetic radiation experiments.

3) The electromagnetic radiation leading to the decline of learning and memory ability of living organisms in radiation verifies the influence of electromagnetic radiation on nervous system.

\section{REFERENCES}

[1] Reisberg B, Ferris SH. \& de LeonMJ, et al. 1982. The global deterioration scale for assessment of primary degenerative dementia, Am. J. Psychiatry, (139): 1136-1139.

[2] Yuan, X.Y. 2010. A brief discussion on electromagnetic compatibility and electromagnetic radiation, China Science and Technology Review, (2): 12-15.

[3] Hutton M. 2000. Molecular genetics of chromosome 17 tauopathies, Ann NY Acad Sci, (920): 63-73.

[4] Ingram EM. \& Spillantini MG. 2002. Tau gene mutations: dissecting the pathogenesis of FTDP-17, Trends Mol Med, (8): 555-562.
[5] Li, L. 2012. The Influence of Electromagnetic Radiation on the Protein Phosphorylation of Primarily Cultured Cortical Neuron tau and A Brief Discussion on Its Mechanism. Beijing: Third Military Medical University.

[6] Gomez-Isla T, Hollister R. \& West H, et al. 1997. Neuronal loss correlates with but exceeds neurofibrillary tangles in Alzheimer's disease, Ann Neurol. (41): 17-24.

[7] Hutton M. 2001. Missense and splice site mutations in tau associated with FTDP-17: multiple pathogenic mechanisms, Neurology. (56): 21-25.

[8] Zhou, H.M., Su, Z.T., Hu, X.J., Xie, X.D., Qu, D.C., Ning, J. \& Yang, G.S. 2009. The calculation of SAR of human central nervous system under the electromagnetic radiation of $50 \mathrm{MHz} 3 \mathrm{GHz}$, Bulletin of the Academy of Military Medical Sciences, 33(1): 57-61.

[9] Lu, G.B., Li, Y.H., Shi, C.H., Xu, Q. \& Liu, S.C. 2013. The influence of $2000 \mu \mathrm{W} / \mathrm{cm} 2$ electromagnetic radiation on the expression of NR2B protein and mRNA of mice hippocampus, Modern Preventive Medicine, 40(11): 2089-2093.

[10]Li, Y.Z., Zhao, K.F., Gui, Y., Li, P., Fang, S.X. \& Ma, Z.J. 2013. The influence of electromagnetic radiation on body physiological indexes, Chinese Journal of Radiological Health, 22(1): 49-51.

[11]Dai, A.H. \& Sun, W.J. 2014. The influence of NADPH oxidase in biological effects of electromagnetic radiation, Progress in Biochemistry and Biophysics, 41(12): 1222-1227.

[12]Zhang, Q., Dai, F.Y., Zang, J. \& Xing, Y.M. 2014. The influence of electromagnetic radiation of different intensity on neurobehavioral functions of $4 \sim 6$ years old children, Occupation and Health, 30(3): 384-387.

[13] Yang, X.X., Li, S.L., Wei, P. \& Feng, L.H. 2014. Electromagnetic wave pollution and hazards, Frontier Science (Quarterly), 29(8): 13-26.

[14]Cheng, F. 2015. A discussion on electromagnetic radiation pollution, 40(1): 126-127.

[15]Zhao, J.S. 1997. Electromagnetic Field and Wave. Xi'an: University of Electronic Science and Technology of China Press.

[16]Gao, J.P. \& Zhang, Z.X. 2002. Electromagnetic Wave Propagation. Xi'an: Northwestern Polytechnical University Press.

[17]XFDTD Reference Manual Version 6.3: 8-16.

[18] Gao, B.Q. 1995. Finite-Difference Time-Domain Method (1st edition). Beijing: National Defense Industry Press.

[19]K.S. Kunz, R.J. Luebber. 1993. The Finite-Difference Time-Domain Method in Electromagnetics, CRC Press.

[20]Yee K S. 1996. Numerical solution of initial boundary value problems involving Maxwell equations in isotropic media, IEEE Trans. Antennas Propagation, AP-14(3): 302-307.

[21]Zhou, W.B., Zhang, X., Yang, X.D., et al. 2007. The calculation of electromagnetic field in simulative dispersion media with Z-transformation FDTD method, $A p$ plication Technology, 34(4): 5-8.

[22]Agilent Technologies EMC Series Analyzers Users Guide. 
ICETA 2015

[23]Li, F.K. \& Wang, Y.C. 2007. Experimental Animals and Animal Experiment Methodology (1st edition). Zhengzhou: Zhengzhou University Press.

[24]Xie, J.T., Liu, Y.Q. \& Cui, G.Y. 2009. Physiology Laboratory (3rd edition). Beijing: Higher Education Press. 